\title{
Circ-VPS13C enhances cisplatin resistance in ovarian cancer via modulating miR-106b-5p/YWHAZ axis
}

\section{Type}

Research paper

\section{Keywords}

cisplatin, Ovarian cancer, circ-VPS13C, miR-106b-5p, YWHAZ

\begin{abstract}
Introduction

Ovarian cancer $(\mathrm{OC})$ is the malignant tumor with the highest mortality among gynecological cancers. Chemotherapy resistance is a major obstacle to OC therapy. Circular RNAs (circRNAs) play crucial roles in cancer development and chemoresistance. However, the role and potential mechanism of has-circ-001567 (circ-VPS13C) in chemoresistance of OC remain unknown.
\end{abstract}

Material and methods

The levels of circ-VPS13C, miR-106b-5p and 14-3-3 zeta (YWHAZ) were detected by quantitative real-time polymerase chain reaction (qRT-PCR) or western blot assay. Cell Counting Kit-8 (CCK-8) assay was used to assess cell viability and calculate the half inhibition concentration (IC50) of cisplatin (DDP). The levels of autophagy-related markers were measured by western blot assay. Cell apoptosis and migration were evaluated by flow cytometry and transwell assay, respectively. The binding relationship between miR-106b-5p and circ-VPS13C or YWHAZ was confirmed by dualluciferase reporter assay. Xenograft assay was performed to explore the role of circ-VPS13C in vivo.

\section{Results}

Circ-VPS13C and YWHAZ were up-regulated, while miR-106b-5p was down-regulated in DDPresistant OC tissues and cells. Knockdown of circ-VPS13C enhanced DDP sensitivity by repressing autophagy in DDP-resistant cells. Circ-VPS13C increased DDP resistance via sponging miR-106b-5p. Moreover, miR-106b-5p directly targeted YWHAZ. Up-regulation of YWHAZ alleviated the decrease in DDP resistance caused by circ-VPS13C depletion. In addition, circ-VPS13C silencing decreased DDP resistance in vivo.

\section{Conclusions}

Circ-VPS13C knockdown enhanced DDP sensitivity of OC through modulation of autophagy via the miR-106b-5p/YWHAZ axis, providing a new biomarker for improving the efficacy of OC

chemotherapy. 
1 Circ-VPS13C enhances cisplatin resistance in ovarian cancer via modulating miR-

$2 \quad 106 b-5 p / Y W H A Z$ axis

3

4 Hairong Yao, Dantong Liu, Fangyuan Gao, Qian Li, Shikai Liu*

5 Department of Gynecology, Cangzhou Central Hospital, Cangzhou City, Hebei

6 Province, China

$7 \quad$ *Corresponding author: Shikai Liu, Department of Gynecology, Cangzhou Central

8 Hospital, No. 16 Xinhua West Road, Yunhe District, Cangzhou 061000, Hebei, China.

$9 \quad$ Tel: +86-182-33739102, E-mail: ojsfg6@163.com

10

11 Running title: The role of circ-VPS13C.

12

13

14

15

16

17 


\section{Abstract}

Background: Ovarian cancer (OC) is the malignant tumor with the highest mortality among gynecological cancers. Chemotherapy resistance is a major obstacle to OC therapy. Circular RNAs (circRNAs) play crucial roles in cancer development and chemoresistance. However, the role and potential mechanism of has-circ-001567 (circVPS13C) in chemoresistance of OC remain unknown.

Methods: The levels of circ-VPS13C, miR-106b-5p and 14-3-3 zeta (YWHAZ) were detected by quantitative real-time polymerase chain reaction (qRT-PCR) or western blot assay. Cell Counting Kit-8 (CCK-8) assay was used to assess cell viability and calculate the half inhibition concentration $\left(\mathrm{IC}_{50}\right)$ of cisplatin (DDP). The levels of autophagyrelated markers were measured by western blot assay. Cell apoptosis and migration were evaluated by flow cytometry and transwell assay, respectively. The binding relationship between miR-106b-5p and circ-VPS13C or YWHAZ was confirmed by dual-luciferase reporter assay. Xenograft assay was performed to explore the role of circ-VPS13C in vivo.

Results: Circ-VPS13C and YWHAZ were up-regulated, while miR-106b-5p was down-regulated in DDP-resistant OC tissues and cells. Knockdown of circ-VPS13C enhanced DDP sensitivity by repressing autophagy in DDP-resistant cells. CircVPS13C increased DDP resistance via sponging miR-106b-5p. Moreover, miR-106b$5 p$ directly targeted YWHAZ. Up-regulation of YWHAZ alleviated the decrease in DDP resistance caused by circ-VPS13C depletion. In addition, circ-VPS13C silencing decreased DDP resistance in vivo.

Conclusion: Circ-VPS13C knockdown enhanced DDP sensitivity of OC through modulation of autophagy via the miR-106b-5p/YWHAZ axis, providing a new biomarker for improving the efficacy of OC chemotherapy.

Keywords: Ovarian cancer, circ-VPS13C, miR-106b-5p, YWHAZ, cisplatin

\section{Background}

Ovarian cancer (OC) is the most common gynecological malignancy with high mortality [1]. Due to atypical symptoms in the early stages of OC, most OC patients 
are diagnosed as advanced stages [2]. Therefore, the prognosis of OC is poor, with a five-year survival rate of about $47 \%$ [3]. Cisplatin, a DNA damage agent, is one of the effective chemotherapy drugs for $\mathrm{OC}$ treatment, but the emergence of cisplatin resistance has become an important factor affecting tumor recurrence [4]. The dysregulation of autophagy is closely related to tumorigenesis and drug resistance [5]. Microtubule-associated protein light chain 3 (LC3) and p62 are typical markers of autophagy, indicating the process of autophagy $[6,7]$. Also, autophagy is characterized by the conversion of LC3-I to LC3-II and the formation of autolysosomes [8]. Therefore, elucidating the molecular mechanism of chemotherapy resistance is essential to improve the prognosis of OC patients.

Circular RNAs (circRNAs) are a new class of non-coding RNAs with stable covalent closed-loop structures [9]. Emerging evidence corroborated that circRNAs exert a crucial regulatory role in the biological and pathological processes of various cancers [10]. Moreover, recent researches revealed that circRNAs could serve as miRNA sponges to regulate tumor progression [11]. For example, circWHSC1 facilitated tumorigenesis in OC via adsorbing microRNA-145 and microRNA-1182 to elevate MUC1 and hTERT expression [12]. CircPUM1 could sponge microRNA-615-5p and microRNA-6753-5p to accelerate OC progression [13]. Also, circRNA Cdr1as potentiated cisplatin sensitivity in OC by sponging microRNA-1270 and increasing SCAI expression [14]. Besides, the previous study suggested that has-circ-001567 (circ-VPS13C) was dramatically up-regulated in OC tissues and cells and was related to poor prognosis [15]. Nevertheless, the effect of circ-VPS13C on cisplatin resistance in OC remains unknown.

MicroRNAs (miRNAs) are highly conserved non-coding RNAs with 18-25 nucleotides. MiRNAs play vital regulatory roles in tumor development and chemoresistance in OC [16]. For instance, Zhang et al. disclosed that miR-1294 overexpression increased sensitivity to cisplatin in OC via inhibiting IGF1R [17]. Lv et al. suggested that miRNA-34a targeted HDAC1 to diminish cisplatin resistance in OC [18]. Weiner-Gorzel et al. revealed that miR-433 strengthened paclitaxel resistance in OC by driving cellular senescence [19]. Additionally, recent researches indicated that 
miR-106b-5p was a tumor-suppressing factor with low expression in OC [20]. However, the role of miR-106b-5p in chemotherapy resistance of OC remains to be investigated.

14-3-3 zeta (YWHAZ) is a member of the 14-3-3 protein family, which can promote cell viability and metastasis in a variety of cancers [21]. Besides, YWHAZ was identified as a biomarker for improving chemoresistance in many types of tumors and a promising prognostic marker for cancer recurrence [22]. Previous studies certified that YWHAZ expression was strikingly increased in cisplatin-resistant $\mathrm{OC}$ cells, and YWHAZ silencing enhanced the sensitivity of OC cells to cisplatin [23].

Hence, we investigated the effect of circ-VPS13C on cisplatin resistance in OC and explored whether it was related to autophagy. In addition, we elucidated the potential molecular mechanism of circ-VPS13C, miR-106b-5p and YWHAZ in OC.

\section{Materials and Methods}

\section{Clinical specimens}

Sixty OC tissues were collected from OC patients who received cisplatin chemotherapy at Cangzhou Central Hospital. The research was ratified by the Ethics Committee of Cangzhou Central Hospital. All participants signed written informed consent. The patients were classified into cisplatin-sensitive $(n=30)$ and cisplatin-resistant $(n=30)$ groups based on response assessment criteria.

\section{Cell culture}

Human ovarian epithelial cell line HOSEpiC and ovarian cancer cell lines (SKOV3 and A2780) were obtained from Shanghai Honsun Biological Technology Co., Ltd (Shanghai, China). SKOV3 and A2780 parental cell lines were continuously exposed to increasing doses of cisplatin (DDP; Solarbio, Beijing, China) to establish DDPresistant OC cell lines (SKOV3/DDP and A2780/DDP). All cells were maintained in RPMI-1640 medium (Gibco, Carlsbad, CA, USA) supplemented with 10\% fetal bovine serum (FBS; Gibco).

\section{Cell transfection}


Small interference RNA (siRNA) against circ-VPS13C (si-circ-VPS13C\#1 and si-circVPS13C\#2), siRNA negative control (si-NC), miR-106b-5p mimics (miR-106b-5p), the mimics control (miR-NC), circ-VPS13C overexpression vector (circ-VPS13C) and the control (pcD5-ciR), YWHAZ overexpression vector (YWHAZ) and the control (pcDNA), miR-106b-5p inhibitor (anti-miR-106b-5p) and the inhibitor control (antimiR-NC) were synthesized from RiboBio (Guangzhou, China). When cell confluence reached approximately $70 \%$, plasmids or oligonucleotides were transfected into DDPresistant OC cells using Lipofectamine 3000 (Invitrogen, Carlsbad, CA, USA).

\section{Quantitative real-time polymerase chain reaction (qRT-PCR)}

The complementary DNA (cDNA) was synthesized using PrimeScript ${ }^{\mathrm{TM}}$ RT Master Mix (Takara, Dalian, China) or miRNA 1st Strand cDNA Synthesis Kit (Vazyme, Nanjing, China) after extracting RNA using Trizol reagent (Invitrogen). Next, quantitative PCR was performed using AceQ qPCR SYBR Green Master Mix (Vazyme). The expression of circ-VPS13C and YWHAZ was normalized by glyceraldehyde-3-phosphate dehydrogenase (GAPDH). MiR-106b-5p expression was normalized by U6. The primers were displayed below: circ-VPS13C (F, 5'TATAATTTTGTCTGCTTCATTTA-3';

$\mathrm{R}$, $5^{\prime}-$ TTAACACAGTCTAAAGTCTCAGAA-3'), miR-106b-5p (F, 5'TGCGGCAACACCAGTCGATGG-3'; R， 5'-CCAGTGCAGGGTCCGAGGT-3'), YWHAZ (F, 5'-TGTAGGAGCCCGTAGGTCATC-3'; R, 5'GTGAAGCATTGGGGATCAAGA-3'), GAPDH (F, 5'ACAACTTTGGTATCGTGGAAGG-3'; R， 5'-GCCATCACGCCACAGTTTC-3'), U6 (F, 5'-CTCGCTTCGGCAGCACA-3'; R, 5'-AACGCTTCACGAATTTGCGT-3').

\section{Cell Counting Kit-8 (CCK-8) assay}

Cells $\left(2 \times 10^{3}\right)$ were seeded into 96-well plates and then stimulated with increasing doses of DDP (0-80 $\mu \mathrm{M})$ for $24 \mathrm{~h}$. Subsequently, the fresh medium containing 10\% CCK-8 solution (Beyotime, Shanghai, China) was added into each well. After incubation for $3 \mathrm{~h}$, cell viability was assessed by measuring optical density at $450 \mathrm{~nm}$ using a 
microplate reader (BioTek, Burlington, VT, USA). The half inhibition concentration $\left(\mathrm{IC}_{50}\right)$ is the dose of DDP at $50 \%$ cell viability.

\section{Western blot assay}

The protein was extracted using RIPA buffer (Solarbio) containing PMSF protease inhibitor (Solarbio), and then quantified using the BCA Protein Assay Kit (Pierce, Appleton, WI, USA). After centrifugation and quantification, equal amounts of protein were separated by polyacrylamide gel electrophoresis and transferred to polyvinylidene fluoride (PVDF) membranes (Millipore, Billerica, MA, USA). Subsequently, the membranes were probed with primary antibodies against LC3B (ab48394, Abcam, Cambridge, UK), P62 (ab155686, Abcam), YWHAZ (ab51129, Abcam) and GAPDH (ab9485, Abcam) after blocking with 5\% skim milk. Next, the membranes were interacted with the secondary antibody (ab7090, Abcam). The intensity of protein was measured using an enhanced chemiluminescence system (Millipore).

\section{Flow cytometry}

After stimulation with $20 \mu \mathrm{M}$ cisplatin for $24 \mathrm{~h}$, the treated cells were plated in six-well plates and resuspended in PBS. AnnexinV-fluorescein isothiocyanate (AnnexinVFITC)/Propidium Iodide (PI) Apoptosis Detection kit (Invitrogen) was utilized to detect cell apoptosis. Also, the apoptosis rate was monitored using BD FACS Calibur flow cytometer (BD Biosciences, San Diego, CA, USA).

\section{Transwell assay}

Treated or untreated cells were seeded in the upper chamber. After $24 \mathrm{~h}$ of incubation, the non-migrating cells were wiped off with a cotton swab, and the migrating cells were stained with crystal violet. Then, the migrated cells were photographed and counted using a microscope.

\section{Dual-luciferase reporter assay}

The sequences of circ-VPS13C or YWHAZ 3'UTR carrying the miR-106b-5p wild 
binding sites were cloned into pmirGLO vector (Promega, Madison, WI, USA) to construct WT-circ-VPS13C or WT-YWHAZ 3'UTR reporter. In addition, MUT-circVPS13C or MUT-YWHAZ 3'UTR reporter containing the miR-106b-5p mutant binding sites was synthesized. Subsequently, the corresponding luciferase reporter and miR-106b-5p or miR-NC were co-transfected into SKOV3/DDP and A2780/DDP cells. Finally, the luciferase intensity was examined using a Dual Luciferase Reporter Assay Kit (Vazyme).

\section{Xenograft assay}

Lentiviral vectors harboring circ-VPS13C short hairpin RNA (sh-circ-VPS13C) or negative control (sh-NC) were purchased from Genelily BioTech (Shanghai, China). Then, SKOV3/DDP cells stably knocked down circ-VPS13C were transfected with miR-106b-5p inhibitor (anti-miR-106b-5p; $50 \mathrm{nM}$ ) or YWHAZ overexpression vector (YWHAZ; $2 \mu \mathrm{g}$ ) using Lipofectamine 3000 (Invitrogen) and selected with $2 \mu \mathrm{g} / \mathrm{mL}$ puromycin for 2 weeks. BALB/c nude mice (five-week-old) were randomly divided into four groups ( $n=7$ per group). SKOV3/DDP cells $\left(5 \times 10^{6}\right)$ transfected with sh-NC, sh-circ-VPS13C, sh-circ-VPS13C+anti-miR-106b-5p or sh-circ-VPS13C+YWHAZ were subcutaneously injected into the right-back of nude mice. After 7 days, the mice were given $5 \mathrm{mg} / \mathrm{kg}$ DDP (Solarbio) every 3 days, and tumor volume was monitored. After 21 days of administration, the mice were sacrificed, and the xenografts were weighted. The expression of circ-VPS13C, miR-106b-5p and YWHAZ was examined by qRT-PCR or western blot. The animal experiment was performed in the Cangzhou Central Hospital and mice were killed by cervical dislocation after deep anesthesia with $2 \%$ isoflurane. The xenograft experiment was approved by the Animal Research Committee of Cangzhou Central Hospital.

\section{Statistical analysis}

All data were shown as mean \pm standard deviation using Graphpad Prism 7.0 software (GraphPad, San Diego, CA, USA). Student's $t$-test was employed to compare differences between two groups, and one-way analysis of variance was used to evaluate 
differences among multiple groups. When $P<0.05$, the difference was considered statistically significant.

\section{Results}

\section{Circ-VPS13C was highly expressed in DDP-resistant OC tissues and cells}

To investigate the potential role of circ-VPS13C in OC, we first examined the expression of circ-VPS13C in tumor tissues of OC patients receiving DDP treatment. The results suggested that circ-VPS13C expression was prominently higher in the resistant group than that in the sensitive group (Fig. 1A). Besides, the expression of circ-VPS13C was remarkably reduced in HOSEpiC cells, while circ-VPS13C expression was markedly increased in SKOV3/DDP and A2780/DDP cells compared to SKOV3 and A2780 cells (Fig. 1B and 1C). From these results, it can be speculated that circ-VPS13C may play a crucial role in DDP resistance of OC. Furthermore, DDPresistant OC cells and parental cells were exposed to increasing concentrations of DDP for $24 \mathrm{~h}$, and cell viability was evaluated by CCK-8 assay. The results revealed that the viability of SKOV3/DDP and A2780/DDP cells was distinctly improved compared with SKOV3 and A2780 cells (Fig. 1D and 1E). Similarly, CCK-8 analysis showed that the $\mathrm{IC}_{50}$ of DDP was drastically higher in SKOV3/DDP and A2780/DDP cells than that in SKOV3 and A2780 cells, suggesting that DDP-resistant cells were successfully constructed (Fig. 1D and 1E). In addition, with the increase of DDP concentration, the ratio of LC3-II/I was gradually increased, and P62 expression was gradually decreased in OC cells (Fig. 1F and 1G). These data suggested that DDP resistance was related to autophagy in OC cells.

\section{Circ-VPS13C knockdown reduced DDP resistance by inhibiting autophagy in}

\section{DDP-resistant OC cells}

To explore the effect of circ-VPS13C on DDP resistance, loss-of-function experiments were performed in DDP-resistant cells transfected with si-NC or si-circ-VPS13C. First of all, the level of circ-VPS13C in the si-circ-VPS13C\#1 group was strikingly decreased compared to the si-NC group, indicating a significant knockdown efficiency 
(Fig. 2A). Therefore, si-circ-VPS13C\#1 was used for subsequent experiments, and sicirc-VPS13C\#1 was simplified as si-circ-VPS13C. CCK-8 assay suggested that silence of circ-VPS13C reduced DDP resistance in SKOV3/DDP and A2780/DDP cells (Fig. 2B). Additionally, circ-VPS13C silencing aggravated cisplatin-inhibited cell viability (Fig. 2C). Also, flow cytometry exhibited that DDP stimulation increased the apoptosis rate of DDP-resistant cells, and the impact was strengthened by inhibiting circ-VPS13C (Fig. 2D). Transwell assay indicated that depletion of circ-VPS13C worsened cisplatinsuppressed cell migration (Fig. 2E). Moreover, DDP treatment led to a marked increase in LC3-II/I level and a significant decrease in P62 expression, while the effects were alleviated after transfection with si-circ-VPS13C\#1 (Fig. 2F). Simultaneously, autophagy inhibitor 3-MA aggravated the inhibition of LC3-II/I expression and the promotion of P62 expression caused by circ-VPS13C silencing (Fig. 2F). These data demonstrated that depletion of circ-VPS13C increased DDP sensitivity through inhibition of autophagy in DDP-resistant cells.

\section{MiR-106b-5p was bound to circ-VPS13C}

To clarify the underlying mechanism of circ-VPS13C, starBase database predicted four target miRNAs (miR-423-5p, miR-106b-5p, miR-152 and miR-30b-3p) that might bind to circ-VPS13C. Subsequently, the expression of four miRNAs was detected by qRTPCR in SKOV3/DDP and A2780/DDP cells transfected with si-NC or si-circ-VPS13C. The results showed that the up-regulation of miR-106b-5p was the most significant after circ-VPS13C knockdown, so miR-106b-5p was selected for follow-up research (Fig. S2A-B). As displayed in Fig. 3A, circ-VPS13C and miR-106b-5p had putative binding sequences. To further validate the prediction, dual-luciferase reporter assay was performed, and the results revealed that mature miR-106b-5p strikingly repressed the luciferase activity of WT-circ-VPS13C reporter in SKOV3/DDP and A2780/DDP cells (Fig. 3B and 3C). Moreover, miR-106b-5p expression was evidently reduced in the resistant group compared with the sensitive group (Fig. 3D). Also, circ-VPS13C level was negatively correlated with miR-106b-5p level in OC tissues (Fig. 3E). Similarly, the expression of miR-106b-5p was remarkably decreased in DDP-resistant OC cells 
relative to parental cells (Fig. 3F). In addition, the overexpression efficiency of circVPS13C was determined by qRT-PCR in SKOV3/DDP and A2780/DDP cells, and the results showed a marked increase in circ-VPS13C expression after transfection with circ-VPS13C overexpression vector (Fig. 3G). Besides, circ-VPS13C silencing enhanced the expression level of miR-106b-5p, and circ-VPS13C up-regulation suppressed miR-106b-5p expression (Fig. 3H). These data evidenced that circ-VPS13C negatively regulated miR-106b-5p in DDP-resistant cells.

\section{Inhibition of miR-106b-5p alleviated the decrease in DDP resistance caused by} circ-VPS13C knockdown in DDP-resistant OC cells

Firstly, miR-106b-5p level was reduced after introduction with anti-miR-106b-5p in DDP-resistant OC cells, indicating a significant inhibition efficiency (Fig. 4A). To investigate whether circ-VPS13C potentiated DDP resistance via modulating miR106b-5p, restoration experiments were carried out to determine this speculation. CCK8 assay exhibited that knockdown of circ-VPS13C caused a distinct decrease in the $\mathrm{IC}_{50}$ of cisplatin, which was attenuated by inhibition of miR-106b-5p (Fig. 4B). Furthermore, DDP treatment and circ-VPS13C depletion conspicuously restrained the viability of DDP-resistant OC cells, while the effect was reversed by inhibiting miR-106b-5p (Fig. 4C). Flow cytometry suggested that DDP stimulation and circ-VPS13C knockdown prominently elevated the apoptosis rate of DDP-resistant OC cells, whereas the impact was abated after transfection with anti-miR-106b-5p (Fig. 4D). Transwell analysis showed that inhibition of miR-106b-5p attenuated the suppressive effect of DDP treatment and circ-VPS13C silencing on cell migration (Fig. 4E). In addition, western blot indicated that DDP stimulation and circ-VPS13C depletion decreased LC3-II/I level and increased P62 expression in SKOV3/DDP and A2780/DDP cells, which were reversed by down-regulating miR-106b-5p (Fig. 4F and 4G). These data demonstrated that circ-VPS13C sponged miR-106b-5p to enhance DDP resistance through regulation of autophagy in DDP-resistant OC cells.

\section{YWHAZ was a target of miR-106b-5p}


Further, the starBase online database showed that miR-106b-5p and YWHAZ 3'UTR had predicted binding sites (Fig. 5A). To confirm whether YWHAZ was a target of miR-106b-5p, dual-luciferase reporter assay was performed. The results displayed that miR-106b-5p overexpression observably reduced the luciferase activity of WTYWHAZ 3'UTR reporter, but had no significant effect on MUT-YWHAZ 3'UTR reporter (Fig. 5B). Additionally, qRT-PCR and western blot results exhibited that the expression of YWHAZ in DDP resistant patients was increased compared to DDP sensitive patients (Fig. 5C and 5D). Spearman's correlation analysis suggested that the levels of circ-VPS13C and YWHAZ were positively correlated in OC tissues (Fig. 5E). Also, the mRNA and protein levels of YWHAZ were markedly elevated in DDPresistant OC cells compared with parental cells (Fig. 5F and 5G). Furthermore, the rescue experiment showed that suppression of miR-106b-5p alleviated the inhibitory effect of circ-VPS13C silencing on YWHAZ protein level (Fig. 5H). Thus, it is concluded that circ-VPS13C regulated YWHAZ expression via sponging miR-106b-5p in DDP-resistant OC cells.

\section{YWHAZ overexpression alleviated the decrease in DDP resistance caused by circ-}

\section{VPS13C knockdown}

First of all, the mRNA and protein levels of YWHAZ were overtly increased after transfection with YWHAZ overexpression vector, suggesting an evident transfection efficiency (Fig. 6A and 6B). To explore whether circ-VPS13C mediated YWHAZ to regulate DDP resistance, SKOV3/DDP and A2780/DDP cells were introduced with siNC, si-circ-VPS13C, si-circ-VPS13C+pcDNA or si-circ-VPS13C+YWHAZ, respectively. CCK-8 assay revealed that up-regulation of YWHAZ reverted the reduction of DDP $\mathrm{IC}_{50}$ caused by circ-VPS13C down-regulation (Fig. 6C). Moreover, functional experiments exhibited that DDP stimulation and circ-VPS13C knockdown impeded cell viability and migration and facilitated apoptosis in DDP-resistant OC cells, whereas YWHAZ overexpression reversed the effects (Fig. 6D-6F). Besides, circVPS13C silencing and DDP treatment hindered autophagy by down-regulating LC3II/I and up-regulating P62, while autophagy was restored by overexpressing YWHAZ 
(Fig. 7G). These data indicated that circ-VPS13C depletion increased DDP sensitivity by regulating YWHAZ through autophagy in DDP-resistant OC cells.

\section{Circ-VPS13C/miR-106b-5p/YWHAZ axis mediated DDP resistance in vivo}

To verify the effect of circ-VPS13C on DDP resistance in vivo, mouse models were established by subcutaneous injection of SKOV3/DDP cells transfected with sh-NC, sh-circ-VPS13C, sh-circ-VPS13C+anti-miR-106b-5p or sh-circ-VPS13C+YWHAZ into BABL/c nude mice. After 7 days of injection, mice were given DDP $(5 \mathrm{mg} / \mathrm{kg})$ every 3 days. The results showed that circ-VPS13C silencing and DDP treatment remarkably decreased tumor volume and weight compared to the sh-NC+DDP group, while these impacts was partially reversed by miR-106b-5p knockdown or YWHAZ overexpression (Fig. 7A-7C). In addition, circ-VPS13C knockdown reduced circVPS13C expression, whereas this influence had not changed after transfection with anti-miR-106b-5p or YWHAZ (Fig. 7D). Moreover, circ-VPS13C silencing elevated miR-106b-5p level and decreased YWHAZ level, and these effects were partially overturned by transfecting anti-miR-106b-5p or YWHAZ (Fig. 7E-7G). These results evidenced that the circ-VPS13C/miR-106b-5p/YWHAZ axis modulated DDP resistance in vivo.

\section{Discussion}

Chemoresistance has been a major stumbling block to chemotherapy in human cancers [24]. Chemotherapy for ovarian cancer mainly depends on cisplatin and paclitaxel [25]. In this study, OC cell resistance to DDP was enhanced through induction of autophagy, which was consistent with previous studies [26, 27]. In addition, we first discovered the function of circ-VPS13C in DDP resistance. In the present study, circVPS13C expression was overtly elevated in OC cells, which was in agreement with previous studies [15]. Moreover, circ-VPS13C was up-regulated in DDP-resistant OC tissues and cells. Loss-of-function experiments demonstrated that circ-VPS13C silencing reduced DDP resistance by suppressing autophagy in DDP-resistant OC cells. Moreover, bioinformatics analysis indicated that miR-106b-5p might be the target of 
circ-VPS13C, which was verified by dual-luciferase reporter assay. Accumulating evidence unveiled that circRNAs could regulate mRNA expression by functioning as competing endogenous RNAs (ceRNAs) of miRNAs [28]. Furthermore, the ceRNA mechanism has been extensively studied in the molecular mechanisms of drug resistance in many cancers. For example, circRNA_0025202 impeded tumor development and increased the sensitivity of tamoxifen in breast cancer by serving as ceRNA for miR-182-5p to up-regulate FOXO3a [29]. Also, circ_0076305 raised the DDP resistance of NSCLC cells by serving as ceRNAs of miR-296-5p to activate STAT3 [30]. Besides, miR-106b-5p has anti-tumor effects in various cancers. Yu et al. revealed that miR-106b-5p increased cisplatin sensitivity in DDP-resistant NSCLC cells by binding to PKD2 [31]. Mastropasqua et al. suggested that miR-17-5p and miR106b-5 targeted TRIM8 to regulate the chemoresistance of anti-tumour drugs in RCC/CRC-derived cells [32]. Moreover, Chen et al. disclosed that miR-106b suppressed the progression of epithelial ovarian cancer via targeting RhoC [33]. In the current research, miR-106b-5p expression was remarkably reduced in DDP-resistant OC tissues and cells. Furthermore, inhibition of miR-106b-5p reversed the decline in DDP resistance induced by circ-VPS13C knockdown via regulating autophagy.

YWHAZ participates in various cellular processes such as apoptosis, metabolism and cell cycle regulation [22]. In hepatocellular carcinoma, YWHAZ accelerated tumor progression via repressing the ubiquitination of heme oxygenase-1 and activating STAT3 signaling [34]. In bladder carcinoma, YWHAZ was strongly associated with tumor invasiveness and increased chemo-/radio-resistance via inhibiting caspaseinduced apoptosis [35]. In addition, YWHAZ potentiated the resistance of OC to DDP and paclitaxel [23]. Similar to recent work, the abundance of YWHAZ was drastically increased in DDP-resistant OC tissues and cells. Interestingly, YWHAZ overexpression reversed the reduction in DDP resistance caused by circ-VPS13C silencing in DDPresistant OC cells.

\section{Conclusion}

In conclusion, circ-VPS13C enhanced cisplatin resistance in ovarian cancer by 
378 inducing autophagy and regulating the miR-106b-5p/YWHAZ axis (Fig. S1). These

379 findings provided new biomarkers for overcoming chemotherapy resistance in OC.

\section{List of abbreviations}

OC: Ovarian cancer

YWHAZ: 14-3-3 zeta

DDP: cisplatin

\section{Disclosure of interest}

The authors declare that they have no financial conflicts of interest.

\section{Acknowledgement}

None.

\section{Funding}

This research was supported by the Cangzhou Key R \& D Program Guidance Project. (Grant No. 183302053)

\section{Ethics approval and consent to participate}

398 The research was ratified by the Ethics Committee of Cangzhou Central Hospital and all participants signed written informed consent.

400

\section{Authors' contributions}

402

Hairong Yao participated in the design of the work, methodology, data interpretation,

403 and drafted the manuscript. Dantong Liu participated in the collection of data and analysis for the work, carried out the statistical analysis. Fangyuan Gao and Qian Li participated in the methodology, data interpretation. Shikai Liu participated in data interpretation and methodology. 
409 All data generated or analyzed during this study are included in this article.

410 


\section{References}

1. Lheureux S, Braunstein M, Oza AM. Epithelial ovarian cancer: Evolution of management in the era of precision medicine. CA Cancer J Clin 2019; 69: 280-304.

2. Singh AP, Senapati S, Ponnusamy MP, Jain M, Lele SM, Davis JS, et al. Clinical potential of mucins in diagnosis, prognosis, and therapy of ovarian cancer. Lancet Oncol 2008; 9: 1076-85.

3. Moufarrij S, Dandapani M, Arthofer E, Gomez S, Srivastava A, Lopez-Acevedo $\mathrm{M}$, et al. Epigenetic therapy for ovarian cancer: promise and progress. Clin Epigenetics 2019; 11: 7 .

4. Damia G, Broggini M. Platinum Resistance in Ovarian Cancer: Role of DNA Repair. Cancers (Basel) 2019; 11.

5. Mele L, Del Vecchio V, Liccardo D, Prisco C, Schwerdtfeger M, Robinson N, et al. The role of autophagy in resistance to targeted therapies. Cancer Treat Rev 2020; 88: 102043.

6. Schaaf MB, Keulers TG, Vooijs MA, Rouschop KM. LC3/GABARAP family proteins: autophagy-(un)related functions. FASEB J 2016; 30: 3961-78.

7. Islam MA, Sooro MA, Zhang P. Autophagic Regulation of p62 is Critical for Cancer Therapy. Int J Mol Sci 2018; 19.

8. Mizushima N, Yoshimori T. How to interpret LC3 immunoblotting. Autophagy 2007; 3: 542-5.

9. White JH, Gallo RM, Bauer WR. Closed circular DNA as a probe for proteininduced structural changes. Trends Biochem Sci 1992; 17: 7-12.

10. Su M, Xiao Y, Ma J, Tang Y, Tian B, Zhang Y, et al. Circular RNAs in Cancer: emerging functions in hallmarks, stemness, resistance and roles as potential biomarkers. Mol Cancer 2019; 18: 90.

11. Ng WL, Mohd Mohidin TB, Shukla K. Functional role of circular RNAs in cancer development and progression. RNA Biol 2018; 15: 995-1005.

12. Zong ZH, Du YP, Guan X, Chen S, Zhao Y. CircWHSC1 promotes ovarian cancer progression by regulating MUC1 and hTERT through sponging miR-145 and miR-1182. J Exp Clin Cancer Res 2019; 38: 437. 
441 13. Guan X, Zong ZH, Liu Y, Chen S, Wang LL, Zhao Y. circPUM1 Promotes 442 Tumorigenesis and Progression of Ovarian Cancer by Sponging miR-615-5p and miR$443 \quad$ 6753-5p. Mol Ther Nucleic Acids 2019; 18: 882-92.

14. Zhao Z, Ji M, Wang Q, He N, Li Y. Circular RNA Cdrlas Upregulates SCAI to Suppress Cisplatin Resistance in Ovarian Cancer via miR-1270 Suppression. Mol Ther Nucleic Acids 2019; 18: 24-33.

15. Bao L, Zhong J, Pang L. Upregulation of Circular RNA VPS13C-has-circ-001567 Promotes Ovarian Cancer Cell Proliferation and Invasion. Cancer Biother Radiopharm 2019; 34: 110-8.

16. Wang Y, Kim S, Kim IM. Regulation of Metastasis by microRNAs in Ovarian Cancer. Front Oncol 2014; 4: 143.

17. Zhang Y, Huang S, Guo Y, Li L. MiR-1294 confers cisplatin resistance in ovarian Cancer cells by targeting IGF1R. Biomed Pharmacother 2018; 106: 1357-63.

18. Lv T, Song K, Zhang L, Li W, Chen Y, Diao Y, et al. miRNA-34a decreases ovarian cancer cell proliferation and chemoresistance by targeting HDAC1. Biochem Cell Biol 2018; 96: 663-71.

19. Weiner-Gorzel K, Dempsey E, Milewska M, McGoldrick A, Toh V, Walsh A, et al. Overexpression of the microRNA miR-433 promotes resistance to paclitaxel through the induction of cellular senescence in ovarian cancer cells. Cancer Med 2015; 4: 74558.

20. Liu Y, Zong ZH, Guan X, Wang LL, Zhao Y. The role of long non-coding RNA PCA3 in epithelial ovarian carcinoma tumorigenesis and progression. Gene 2017; 633: $42-7$.

21. Matta A, Siu KW, Ralhan R. 14-3-3 zeta as novel molecular target for cancer therapy. Expert Opin Ther Targets 2012; 16: 515-23.

22. Neal CL, Yu D. 14-3-3zeta as a prognostic marker and therapeutic target for cancer. Expert Opin Ther Targets 2010; 14: 1343-54.

23. Hong L, Chen $\mathrm{W}$, Xing $\mathrm{A}$, Wu D, Wang S. Inhibition of Tyrosine 3Monooxygenase/Tryptophan 5-Monooxygenase Activation Protein Zeta (YWHAZ) Overcomes Drug Resistance and Tumorigenicity in Ovarian Cancer. Cell Physiol 
Biochem 2018; 49: 53-64.

24. Vasan N, Baselga J, Hyman DM. A view on drug resistance in cancer. Nature 2019; 575: 299-309.

25. Bookman MA. Optimal primary therapy of ovarian cancer. Ann Oncol 2016; 27 Suppl 1: i58-i62.

26. Tan WX, Xu TM, Zhou ZL, Lv XJ, Liu J, Zhang WJ, et al. TRP14 promotes resistance to cisplatin by inducing autophagy in ovarian cancer. Oncol Rep 2019.

27. Zhou F, Yang X, Zhao H, Liu Y, Feng Y, An R, et al. Down-regulation of OGT promotes cisplatin resistance by inducing autophagy in ovarian cancer. Theranostics 2018; 8: 5200-12.

28. Zhong Y, Du Y, Yang X, Mo Y, Fan C, Xiong F, et al. Circular RNAs function as ceRNAs to regulate and control human cancer progression. Mol Cancer 2018; 17: 79. 29. Sang Y, Chen B, Song X, Li Y, Liang Y, Han D, et al. circRNA_0025202 Regulates Tamoxifen Sensitivity and Tumor Progression via Regulating the miR-182-5p/FOXO3a Axis in Breast Cancer. Mol Ther 2019; 27: 1638-52.

30. Dong Y, Xu T, Zhong S, Wang B, Zhang H, Wang X, et al. Circ_0076305 regulates cisplatin resistance of non-small cell lung cancer via positively modulating STAT3 by sponging miR-296-5p. Life Sci 2019; 239: 116984.

31. Yu S, Qin X, Chen T, Zhou L, Xu X, Feng J. MicroRNA-106b-5p regulates cisplatin chemosensitivity by targeting polycystic kidney disease-2 in non-small-cell lung cancer. Anticancer Drugs 2017; 28: 852-60.

32. Mastropasqua F, Marzano F, Valletti A, Aiello I, Di Tullio G, Morgano A, et al. TRIM8 restores p53 tumour suppressor function by blunting N-MYC activity in chemo-resistant tumours. Mol Cancer 2017; 16: 67.

33. Chen S, Chen X, Xiu YL, Sun KX, Zhao Y. Inhibition of Ovarian Epithelial Carcinoma Tumorigenesis and Progression by microRNA 106b Mediated through the RhoC Pathway. PLoS One 2015; 10: e0125714.

34. Song J, Zhang X, Liao Z, Liang H, Chu L, Dong W, et al. 14-3-3zeta inhibits heme oxygenase-1 (HO-1) degradation and promotes hepatocellular carcinoma proliferation: involvement of STAT3 signaling. J Exp Clin Cancer Res 2019; 38: 3. 
35. Yu CC, Li CF, Chen IH, Lai MT, Lin ZJ, Korla PK, et al. YWHAZ amplification/overexpression defines aggressive bladder cancer and contributes to chemo-/radio-resistance by suppressing caspase-mediated apoptosis. J Pathol 2019; 248: $476-87$.

\section{Figure legends}

Fig. 1 Circ-VPS13C was highly expressed in DDP-resistant OC tissues and cells. (A) The expression of circ-VPS13C in OC resistant tissues $(n=30)$ and sensitive tissues $(\mathrm{n}=30)$ was measured using qRT-PCR. (B) The level of circ-VPS13C in HOSEpiC, SKOV3 and SKOV3/DDP cells was detected by qRT-PCR $(n=3)$. (C) The expression of circ-VPS13C in HOSEpiC, A2780 and A2780/DDP cells was examined by qRT-PCR $(n=3)$. (D and E) SKOV3, SKOV3/DDP, A2780 and A2780/DDP cells were stimulated with different concentrations of DDP for $24 \mathrm{~h}$, and CCK-8 analysis was used to detect cell viability and the $\mathrm{IC}_{50}$ of cisplatin $(\mathrm{n}=3)$. ( $\mathrm{F}$ and $\left.\mathrm{G}\right)$ The levels of autophagy-related proteins were measured using western blot assay in SKOV3, SKOV3/DDP, A2780 and A2780/DDP cells treated with varying doses of DDP $(0 \mu \mathrm{M}, 5 \mu \mathrm{M}, 10 \mu \mathrm{M}$ and $20 \mu \mathrm{M})$ $(\mathrm{n}=3)$. Data were presented as mean \pm SD. $* P<0.05$.

Fig. 2 Circ-VPS13C knockdown reduced DDP resistance by inhibiting autophagy in DDP-resistant OC cells. (A and B) SKOV3/DDP and A2780/DDP cells were introduced with si-NC or si-circ-VPS13C. (A) The knockdown efficiency of circVPS13C was determined by qRT-PCR. (B) The $\mathrm{IC}_{50}$ of cisplatin was evaluated by CCK-8 assay. (C-F) SKOV3/DDP and A2780/DDP cells were treated with DDP (20 $\mu \mathrm{M}$ ) for $24 \mathrm{~h}$ after transfection with si-NC or si-circ-VPS13C for $48 \mathrm{~h}$. (C) Cell viability was assessed by CCK-8 assay. (D) Cell apoptosis was detected by flow cytometry. (E) Cell migration was measured by transwell assay. (F) The expression of autophagyrelated proteins was determined by western blot. Data were presented as mean $\pm \mathrm{SD}$, $\mathrm{n}=3 . * P<0.05$. 
Fig. 3 MiR-106b-5p was bound to circ-VPS13C. (A) The starBase predicted that circVPS13C and miR-106b-5p had putative binding sites. (B and C) SKOV3/DDP and A2780/DDP cells were co-transfected with WT-circ-VPS13C or MUT-circ-VPS13C and miR-106b-5p or miR-NC, and the luciferase activity was examined by dualluciferase reporter assay ( $n=3$ ). (D) QRT-PCR was used to detect miR-106b-5p expression in DDP resistant $(n=30)$ and sensitive $(n=30)$ groups. (E) The correlation between circ-VPS13C and miR-106b-5p was tested by Spearman's correlation coefficient (n=30). (F) MiR-106b-5p level was measured in HOSEpiC cells, parental OC cells and DDP-resistant OC cells $(n=3)$. (G) The overexpression efficiency of circVPS13C was detected by qRT-PCR $(n=3)$. (H) The expression of miR-106b-5p was measured by qRT-PCR in SKOV3/DDP and A2780/DDP cells transfected with si-NC, si-circ-VPS13C, pcD5-ciR or circ-VPS13C $(n=3)$. Data were presented as mean $\pm \mathrm{SD}$. $* P<0.05$.

Fig. 4 Inhibition of miR-106b-5p alleviated the decrease in DDP resistance caused by circ-VPS13C knockdown in DDP-resistant OC cells. (A) The inhibition efficiency of miR-106b-5p was detected by qRT-PCR. (B) SKOV3/DDP and A2780/DDP cells were transduced with si-NC, si-circ-VPS13C, si-circ-VPS13C+antimiR-NC or si-circ-VPS13C+anti-miR-106b-5p, and the IC $_{50}$ of cisplatin was measured by CCK-8 assay. (C-G) SKOV3/DDP and A2780/DDP cells were stimulated with DDP $(20 \mu \mathrm{M})$ for $24 \mathrm{~h}$ after introduction with si-NC, si-circ-VPS13C, si-circ-VPS13C+antimiR-NC or si-circ-VPS13C+anti-miR-106b-5p for $48 \mathrm{~h}$. (C) CCK-8 assay was used to assess cell viability. (D) Flow cytometry was utilized to monitor cell apoptosis. (E) Transwell assay was conducted to detect cell migration. ( $F$ and $G$ ) The levels of autophagy-related proteins were measured by western blot. Data were presented as mean $\pm \mathrm{SD}, \mathrm{n}=3 . * P<0.05$.

Fig. 5 YWHAZ was a target of miR-106b-5p. (A) The predicted binding sites of miR106b-5p and YWHAZ 3'UTR was shown. (B) Dual-luciferase reporter assay was conducted to verify the relationship between miR-106b-5p and YWHAZ ( $n=3)$. (C and D) The mRNA and protein levels of YWHAZ were examined in DDP resistant $(n=30)$ and sensitive $(n=30)$ groups by qRT-PCR and western blot. (E) The correlation between 
miR-106b-5p and YWHAZ was detected by Spearman's correlation coefficient $(n=30)$. (F and G) The mRNA and protein levels of YWHAZ were measured in HOSEpiC cells, parental OC cells and DDP-resistant OC cells $(n=3)$. (H) The mRNA and protein expression of YWHAZ was detected in DDP-resistant OC cells transfected with si-NC, si-circ-VPS13C, si-circ-VPS13C+anti-miR-NC or si-circ-VPS13C+anti-miR-106b-5p $(\mathrm{n}=3)$. Data were presented as mean \pm SD. $* P<0.05$.

Fig. 6 YWHAZ overexpression alleviated the decrease in DDP resistance caused by circ-VPS13C knockdown. (A and B) The overexpression efficiency of YWHAZ was detected using qRT-PCR and western blot. (C) SKOV3/DDP and A2780/DDP cells were transfected with si-NC, si-circ-VPS13C, si-circ-VPS13C+pcDNA or si-circVPS13C + YWHAZ, and the $\mathrm{IC}_{50}$ of cisplatin was assessed by CCK-8 assay. (D-G) SKOV3/DDP and A2780/DDP cells were introduced with si-NC, si-circ-VPS13C, sicirc-VPS13C + pcDNA or si-circ-VPS13C + YWHAZ and then stimulated with DDP for 24 h. (D) Cell viability was detected by CCK-8 assay. (E) Cell apoptosis was monitored by flow cytometry. (F) Cell migration was evaluated by transwell assay (G) The levels of LC3-II/I and P62 were examined using western blot. Data were presented as mean \pm $\mathrm{SD}, \mathrm{n}=3 . * P<0.05$.

Fig. 7 Circ-VPS13C/miR-106b-5p/YWHAZ axis mediated DDP resistance in vivo. SKOV3/DDP cells transfected with sh-NC, sh-circ-VPS13C, sh-circ-VPS13C+antimiR-106b-5p or sh-circ-VPS13C+YWHAZ were subcutaneously injected into nude mice. (A and B) After 7 days, $5 \mathrm{mg} / \mathrm{kg}$ of DDP was injected into nude mice every 3 days, and tumor volume was measured $(n=7)$. (C) After 21 days of administration, the mice were killed, and tumor weight was determined ( $n=7)$. (D-G) The levels of circVPS13C, miR-106b-5p and YWHAZ were detected by qRT-PCR and western blot $(\mathrm{n}=3)$. Data were presented as mean \pm SD. $* P<0.05$.

Fig. S1 The schematic diagram showed the mechanism by which circ-VPS13C regulated cisplatin resistance via the miR-106b-5p/YWHAZ axis.

Fig. S2 The expression of four possible target miRNAs (miR-423-5p, miR-106b-5p, miR-152 and miR-30b-3p) was detected using qRT-PCR in SKOV3/DDP and A2780/DDP cells transfected with si-NC or si-circ-VPS13C. Data were presented as 
591 mean $\pm \mathrm{SD}, \mathrm{n}=3 . * P<0.05$.

592 
A

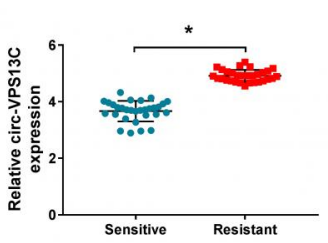

D

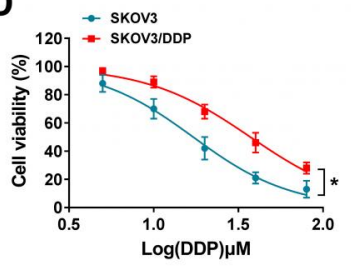

F

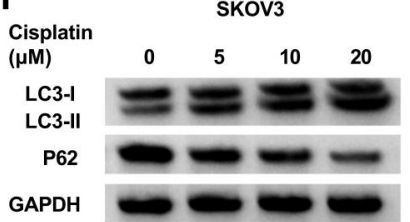

G

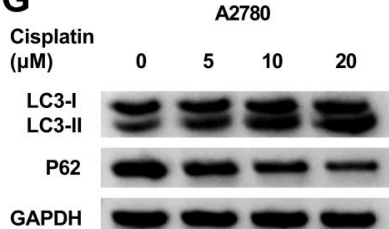

B
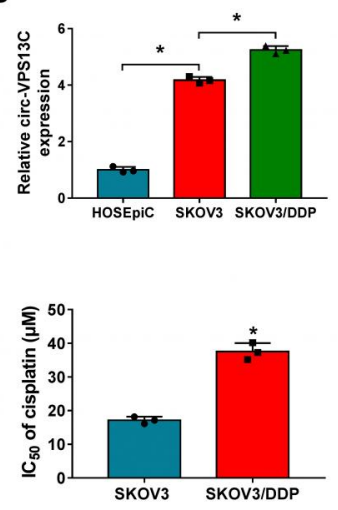

SKOV3/DDP

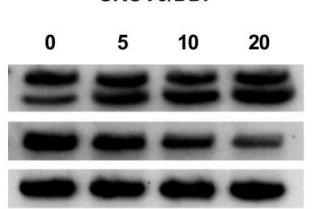

A2780/DDP

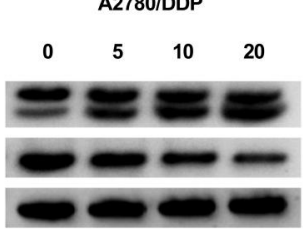

C

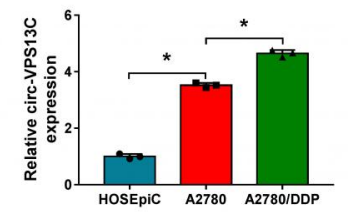

E
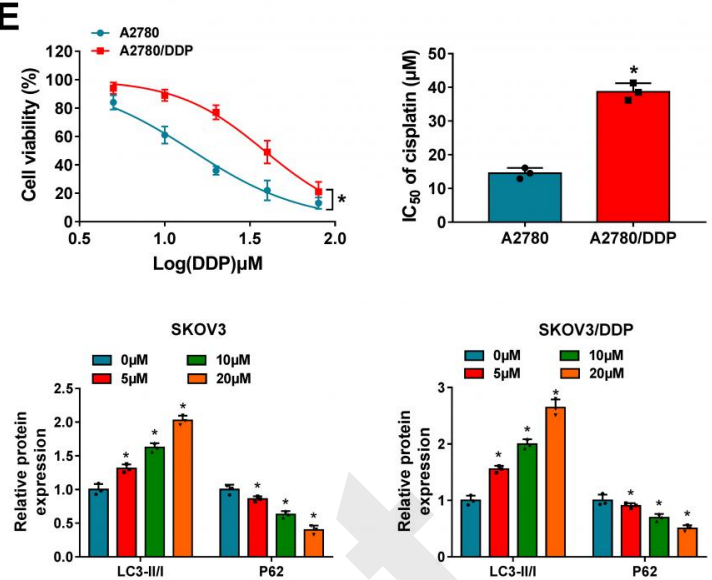
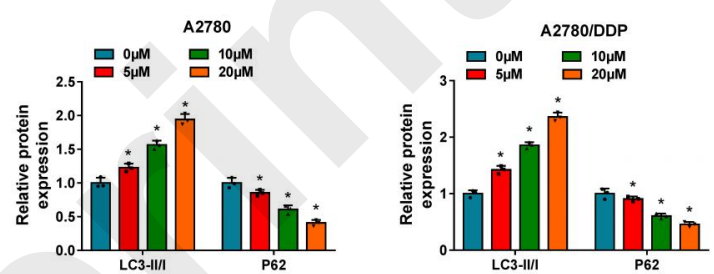
A

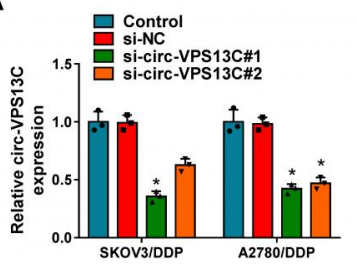

B

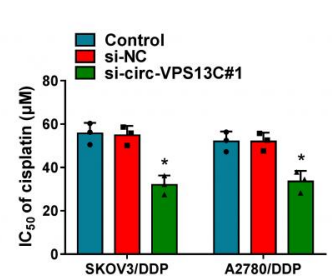

C 昌 Control

DDP+SIINC

${ }^{150}$ 吕

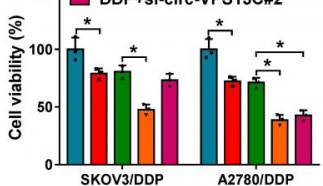

D
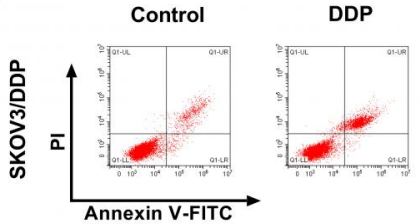

DDP+si-NC DDP+si-circ-VPS13C\#1
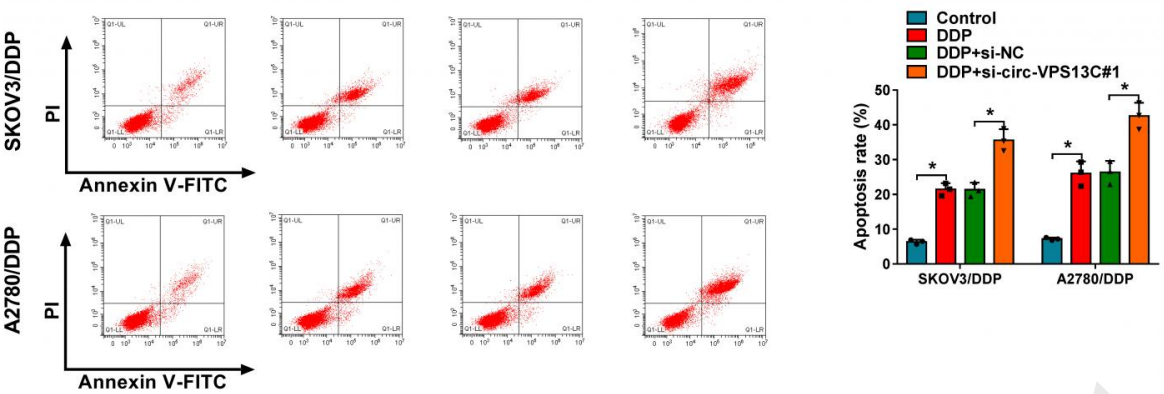

E

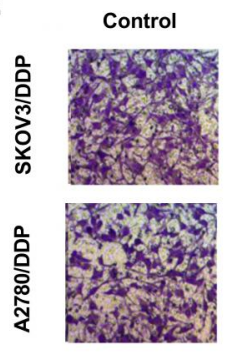

DDP

DDP+si-NC DDP+si-circ-VPS13C\#1
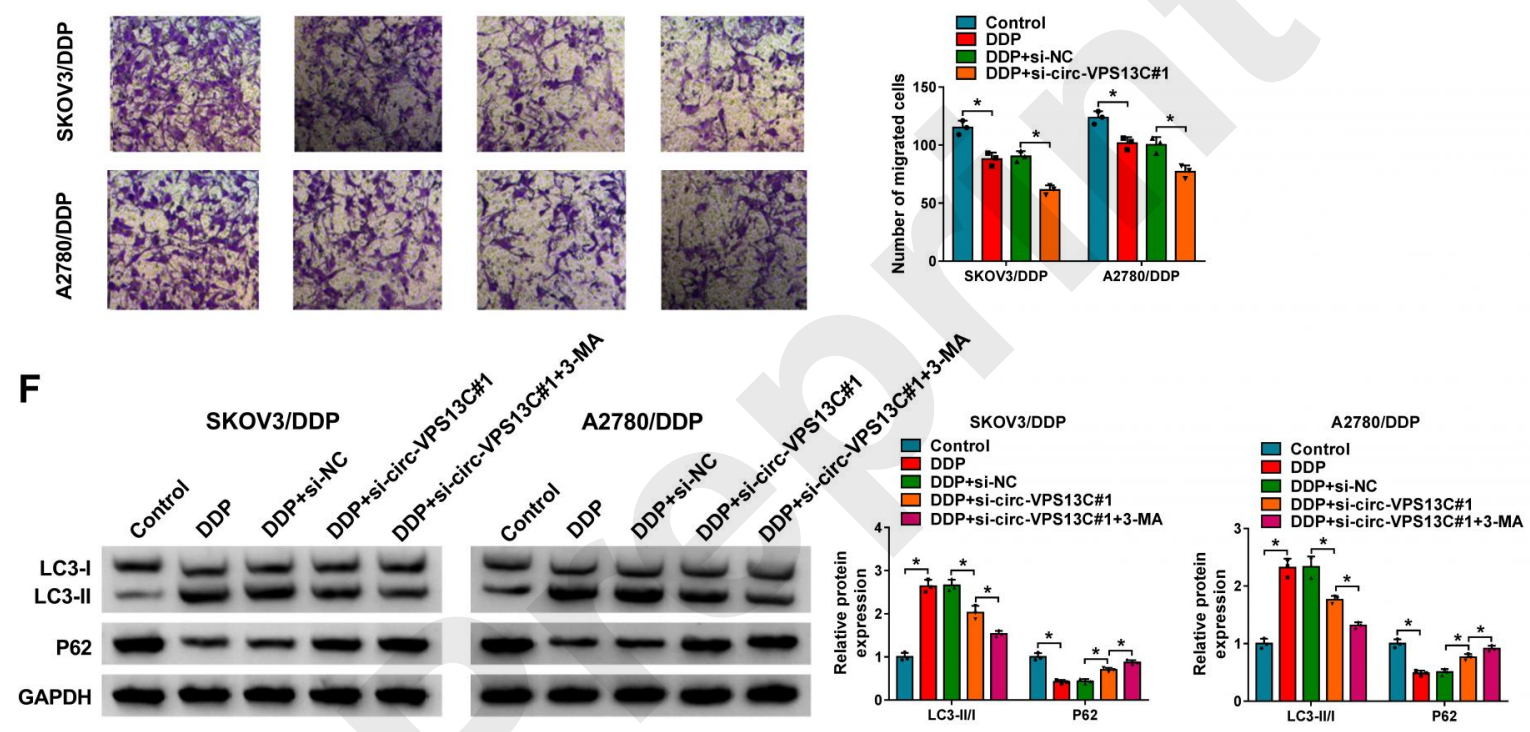
A

circ-VPS13C WT 5' CGACAgGaUAAUGgCACUUUA 3' miR-106b-5p 3' UAGACGUGACAGUCGUGAAAU 5'

circ-VPS13C MUT 5' CGACAGGAUAAUGCGUGAAAA 3'

B

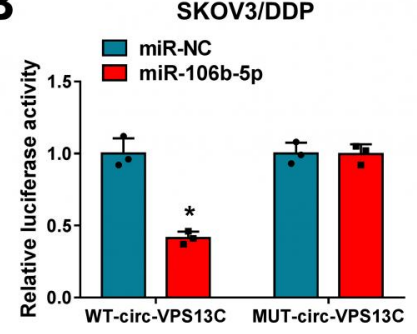

$\mathbf{F}$

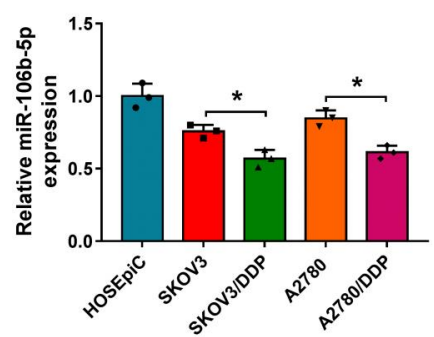

C

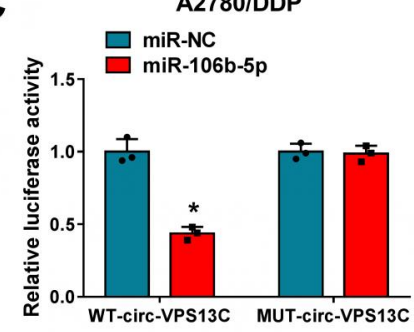

G

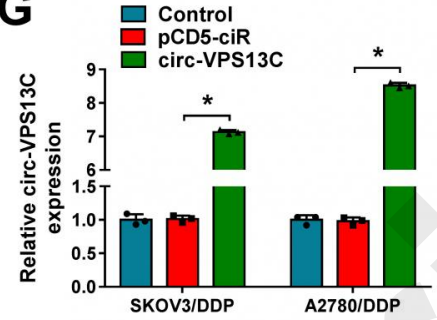

D

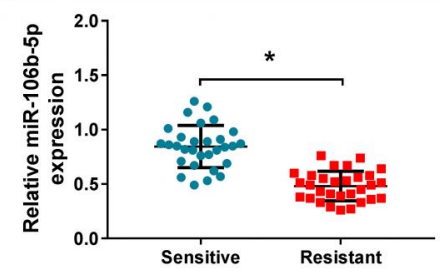

E

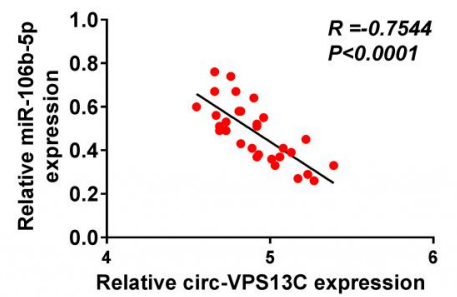

H

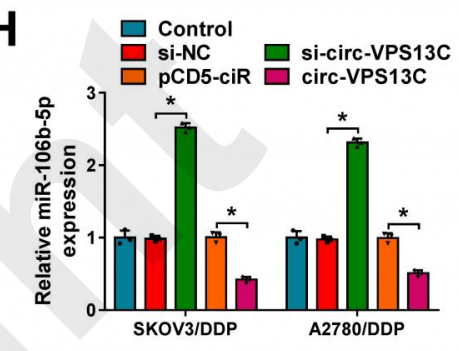




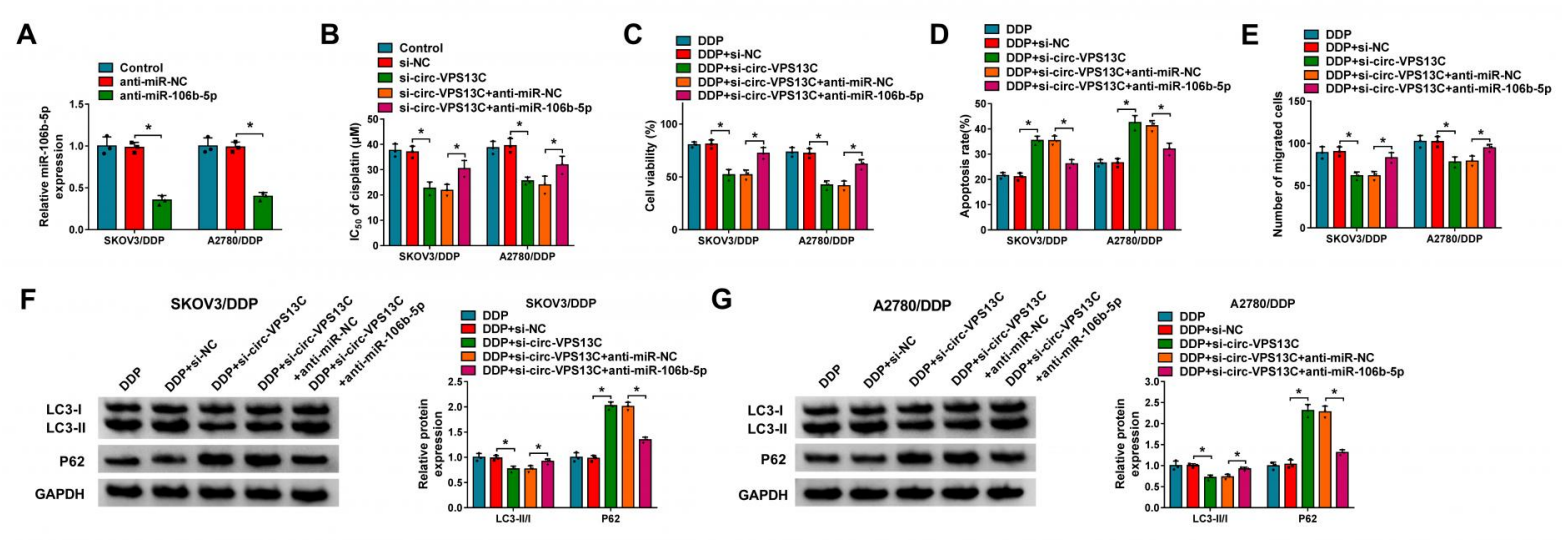


A

YWHAZ 3'UTR WT 5' UUUUGUGCUUCCAAAUCACUUUU $3^{\prime}$ miR-1066-5p 3' UAGACGUGACAG- - UCGUGAAAU 5'

YWHAZ 3'UTR MUT 5' UUUUGUGCUUCCAAAUGUGAAAU 3'
B

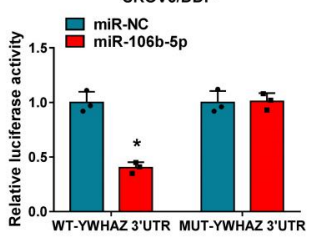

C

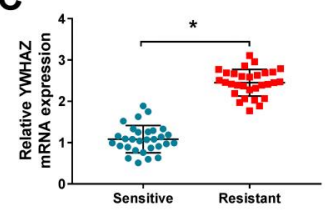

D
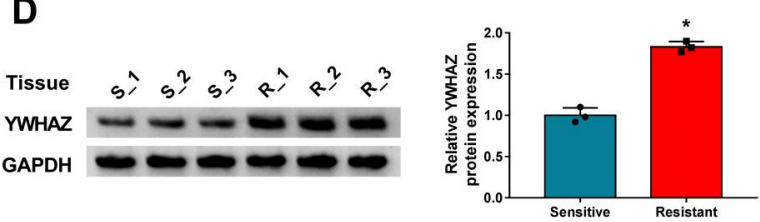

\section{$\mathbf{F}$}

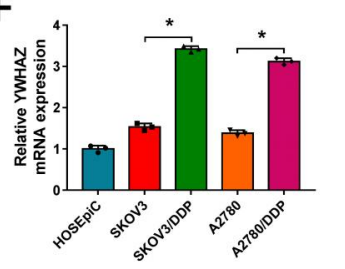

G

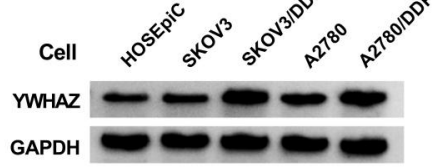

H

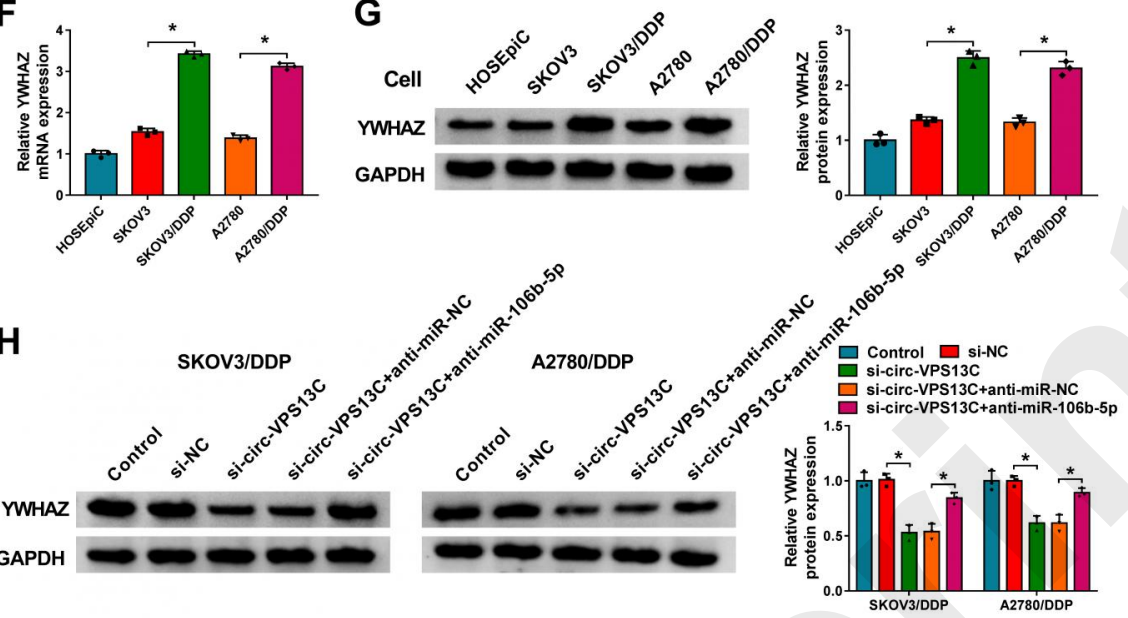

E

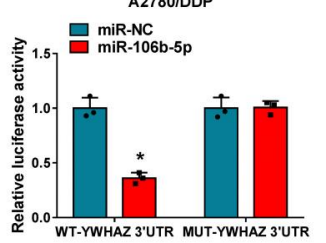

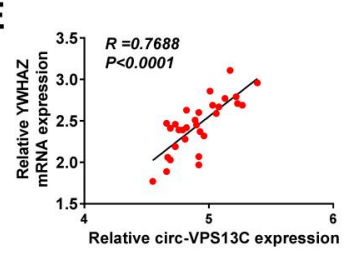




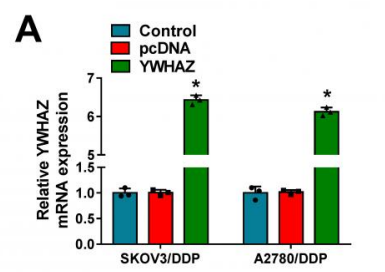

B
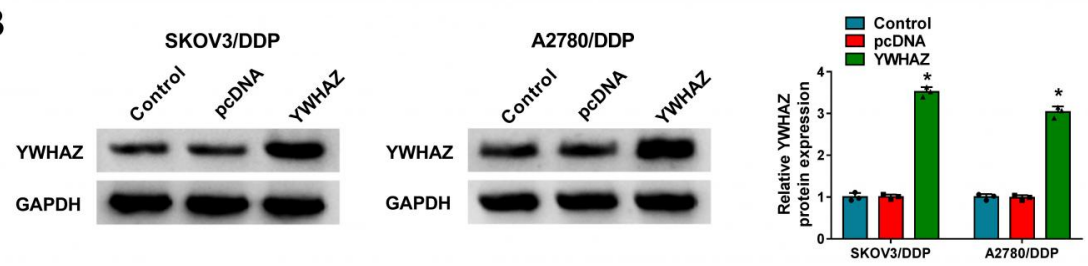

C

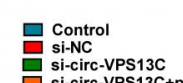

si-circ-VPS13C
si-circ-VPS13C+pcDNA
si-circ-VPS13C+YWHAZ

D

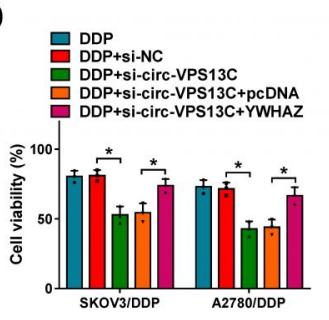

E 昌总p

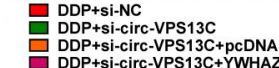

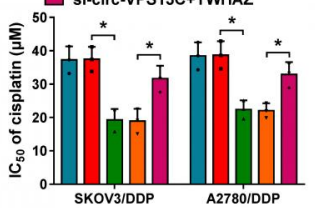

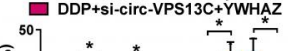

F

旦暨P+sinc

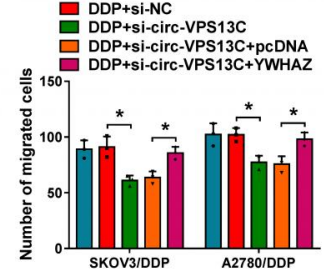

G

SKOV3/DDP

A2780/DDP
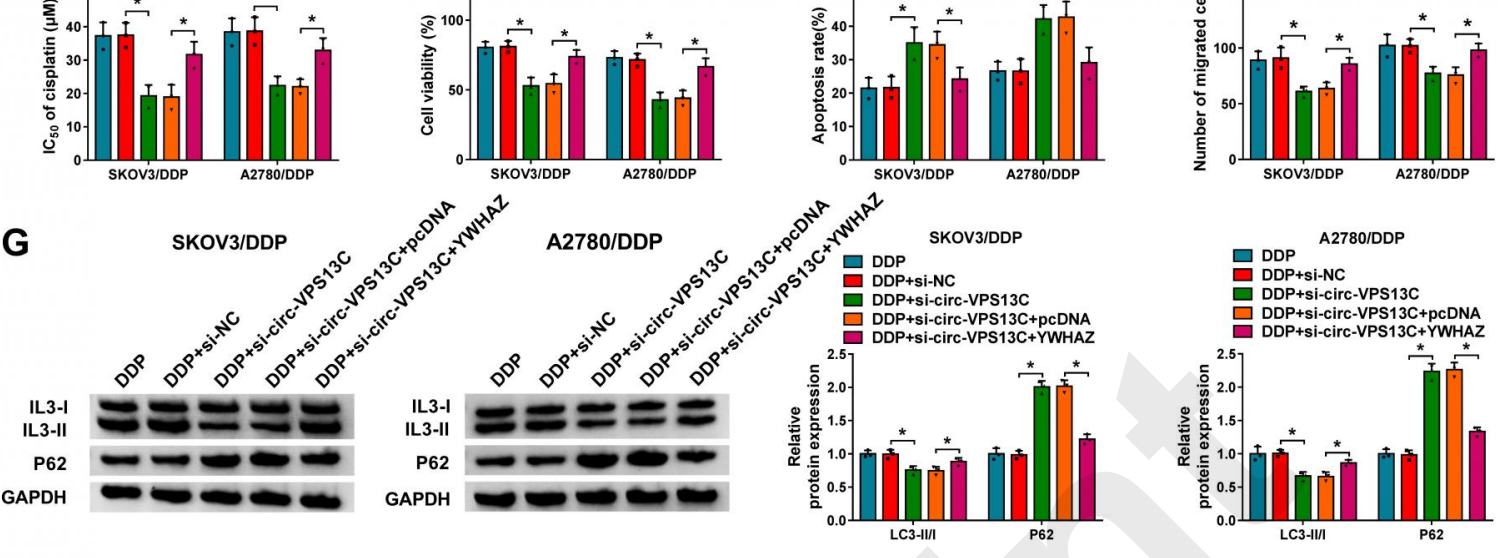

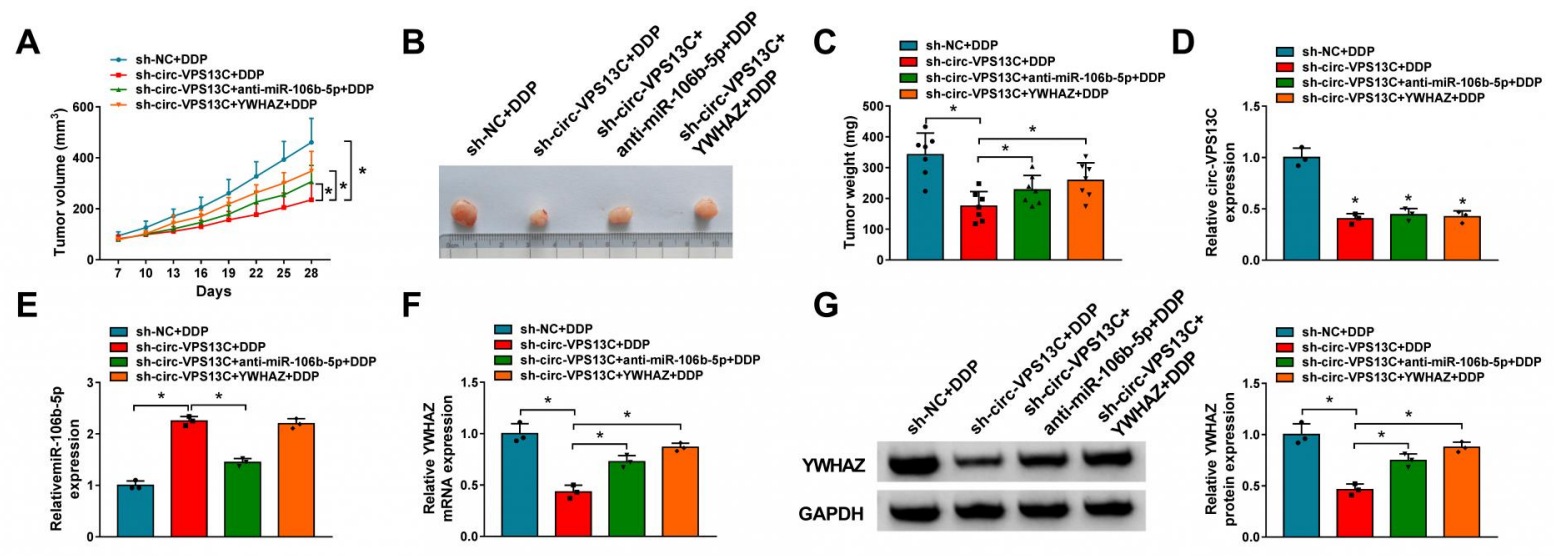


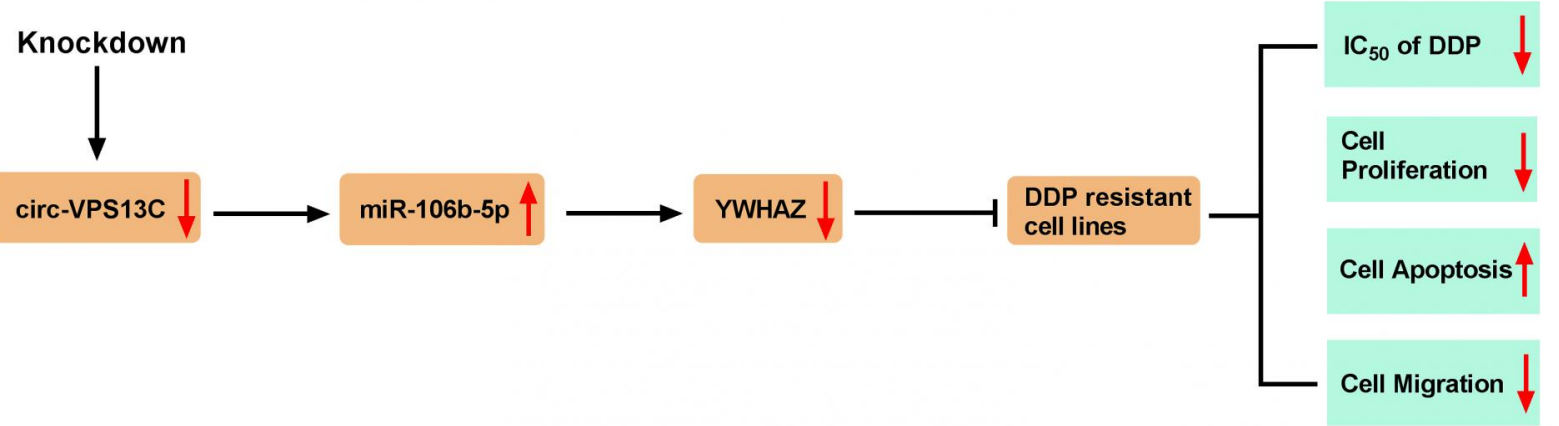


A

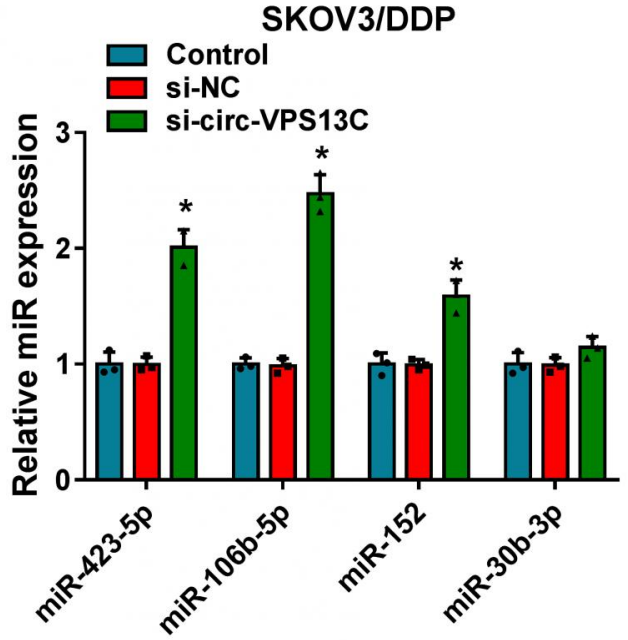

B

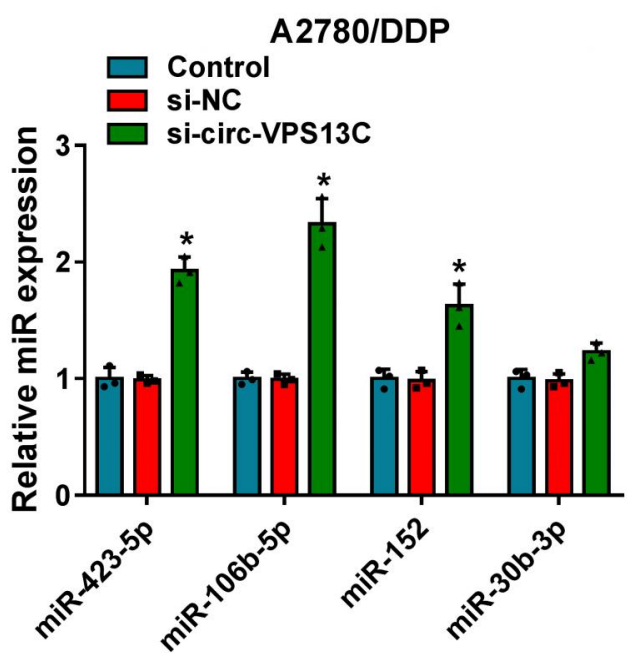

